\title{
Concepção de residentes multiprofissionais sobre gestão de serviços de saúde mental
}

\author{
Zaira Letícia Tisott ${ }^{1}$ \\ Leila Mariza Hildebrandt ${ }^{2}$ \\ Luiz Anildo Anacleto da Silva ${ }^{2}$ \\ Fernanda Beheregaray Cabral ${ }^{2}$ \\ Marinês Tambara Leite ${ }^{2}$
}

\begin{abstract}
Objetivo: apreender a concepção de profissionais de saúde, residentes do Programa de Residência Multiprofissional em Saúde, acerca do processo de Gestão Pública de Serviços de Saúde Mental e compreender a influência do processo de gestão na formação dos profissionais residentes. Método: Pesquisa qualitativa e descritiva, realizada com 13 profissionais residentes. A coleta de dados ocorreu por meio de entrevista semiestruturada. Os dados foram analisados por meio de análise temática. Resultados: Observou-se a importância de o gestor em saúde possuir conhecimentos referentes à saúde mental e às políticas que a regem. Ainda, a participação de residentes na gestão contribui na formação profissional. Conclusão: Conclui-se que a gestão implica diretamente nos processos de trabalho da equipe e dos profissionais residentes.
\end{abstract}

Descritores: Saúde Mental; Serviços Comunitários de Saúde Mental; Gestão em Saúde.

\footnotetext{
${ }^{1}$ Mestranda, Universidade Federal de Santa Maria, Santa Maria, RS, Brasil. Bolsista da Coordenação de Aperfeiçoamento de Pessoal de Nível Superior (CAPES), Brasil.

${ }^{2} \mathrm{PhD}$, Professor Adjunto, Universidade Federal de Santa Maria, Palmeira das Missões, RS, Brasil.
}

\author{
Correspondência: \\ Zaira Letícia Tisott \\ Universidade Federal de Santa Maria \\ Av Roraima, 1000, prédio 26, sala 1445 \\ CEP: 97105-970, Santa Maria, RS, Brasil. \\ E-mail: zairatisott10@gmail.com
}




\section{Conception of multiprofessional residents on the management of mental}

\section{health services}

Objetive: to obtain the conception of professionals of health, residents of Multiprofessional Residence in Health Program, about the process of Public Management of Mental Health Services and to comprehend theinfluence of process of management in the education of resident professionals. Method: Qualitative and descriptive research, produced with 13 resident professionals. The data was collected through a semistructured interview and analyzed by thematic area. Results: It was observed the importance of the manager in health has some knowledge about mental health and the politics that rule them. Still, the participation of residents in management contributes in professional education. Conclusion: It was concluded that the management implicates directly in the process of teamwork and in professional qualification of residents, through lived experiences.

Descriptors: Mental health; Communitarian Services of Health; Management in Health.

\section{Las concepciones de los residentes multiprofesionales en la gestión de servicios de salud men}

Objetivo: comprender el concepto de los profesionales de la salud, los residentes de la Residencia Multiprofesional en Salud, acerca del proceso de Gestión Pública de los Servicios de Salud Mental y comprender la influencia del proceso de gestión en la formación de los profesionales residentes. Método: investigación cualitativa y descriptiva, realizada con 13 profesionales residentes. Los datos fueron recolectados por medio de entrevistas y analizados por medio del análisis temático. Resultados: Se señaló la importancia del gerente en la salud tener los conocimientos relativos a la salud mental y las políticas que lo rigen. Aun así, la participación de los residentes en la gestión contribuye en la formación profesional. Conclusión: Se concluye que la gestión implica directamente en los procesos del trabajo en equipo y en la cualificación profesional de los residentes, de las experiencias vividas.

Descriptores: Salud Mental; Servicios de Salud Mental de la Comunidad; La Gestión de la Salud.

\section{Introdução}

O início da Reforma Psiquiátrica no Brasil se deu em favor da mudança dos modelos de atenção e gestão nas práticas de saúde mental(1-2). Diante disso, a Lei Federal $N^{\circ} 10.216$, sancionada em 2001, elenca os direitos das pessoas com transtorno mental e o fim das práticas manicomiais no País ${ }^{(3)}$. A partir dessa legislação, vários serviços foram estruturados com vistas a atender as demandas dos usuários com transtornos mentais e seus familiares. A Portaria $\mathrm{N}^{\circ}$ 3.088 de 2011 instituiu a Rede de Atenção Psicossocial (RAPS), incluindo diversos serviços, dentre eles os
Centros de Atenção Psicossocial (CAPS) e o Hospital Geral $(\mathrm{HG})^{(4)}$.

Embora várias mudanças tenham sido propostas pela Reforma Psiquiátrica, o Ministério da Saúde tem desenvolvido estratégias voltadas para a formação e qualificação de trabalhadores de saúde com base nos princípios do Sistema Único de Saúde (SUS), dentre elas, a Residência Multiprofissional em Saúde, criada pela Portaria N. ${ }^{\circ} 2.117 / 05^{(5)}$. Esta é organizada e desenvolvida em parceria com gestores, trabalhadores e usuários dos serviços de saúde, visando à integração ensino-serviço-comunidade ${ }^{(6)}$. Nesse contexto, insere-se a Política Nacional de Humanização da Atenção e 
Gestão no Sistema Único de Saúde-HumanizaSUS (PNH), que propõe a promoção de práticas de gestão e de atenção em saúde ${ }^{(7)}$. A gestão em saúde, por vezes, está baseada na avaliação e cumprimento de metas. Entretanto, para enfrentar essa lógica, é necessário levar propostas e ofertas de reorganização dos serviços, como o modelo de cogestão, que prevê decisões coletivas, por meio da constituição de espaços coletivos e do agir comunicativo, a fim de pensar a ação( ${ }^{(8)}$. Nesse sentido, o estudo tem como objetivos, apreender a concepção de profissionais de saúde, residentes do Programa de Residência Multiprofissional em Saúde, acerca do processo de Gestão Pública de serviços de Saúde Mental e compreender a influência do processo de gestão dos serviços de saúde mental na formação dos profissionais residentes.

\section{Metodologia}

Pesquisa qualitativa e descritiva, realizada com todos os profissionais residentes do segundo ano de um Programa de Residência Multiprofissional Integrada em Sistema Público de Saúde (PRMISPS) com ênfase em saúde mental, da região central do Rio Grande do Sul, em um total de 13 profissionais. Os participantes do estudo encontravam-se inseridos em uma Unidade de internação psiquiátrica de hospital geral e em três CAPS, sendo estes, um CAPS II, um CAPS II i (infanto juvenil) e um CAPS II ad (álcool e outras drogas). Dos 13 participantes, três eram Enfermeiras, cinco Psicólogas, quatro Assistentes Sociais e uma Terapeuta Ocupacional, sendo todas as profissionais do gênero feminino.

Com relação à idade, a faixa etária foi de 25 a 36 anos. Em relação à experiência profissional, oito participantes eram recém-formadas, três possuíam experiência de trabalho em outros municípios em suas respectivas áreas de formações, anterior a Residência, duas participantes tinham feito Residência anteriormente, sendo que uma delas possua título de Mestre e duas especializações em Gestão Pública.

A coleta de dados foi realizada nos meses de agosto e setembro de 2015, por meio de entrevistas semiestruturadas. As entrevistas, gravadas por meio digital de áudio e transcritas na íntegra, foram realizadas nas instituições de saúde em que os profissionais residentes desenvolviam suas atividades, em horário previamente combinado.

A técnica escolhida para análise dos dados foi o da Análise Temática ${ }^{(9)}$, a qual consiste em descobrir os núcleos de sentido que compõem uma comunicação, cuja presença ou frequência tem algum significado para o objeto analítico visado. A análise temática desdobra-se em três etapas: a pré-análise, que engloba a escolha dos documentos a serem analisados e a retomada de hipóteses e objetivos da pesquisa; a segunda etapa envolve a exploração do material e sua classificação, com vistas a alcançar o núcleo de compreensão do texto; e a terceira etapa consiste no tratamento dos resultados obtidos e interpretação, em que o pesquisador propõe inferências e realiza interpretações, interrelacionando-as com o quadro teórico que baliza o estudo.

Os princípios éticos da pesquisa foram observados, conforme a Resolução № 466/12 do Conselho Nacional de Saúde, de modo a proteger os direitos dos participantes ${ }^{(10)}$. O projeto de pesquisa foi aprovado pelo Comitê de Ética em Pesquisa da Universidade Federal de Santa Maria/UFSM, mediante Parecer Consubstanciado $\mathrm{N}^{\circ} 1.182 .777$.

\section{Resultados}

A partir da leitura exaustiva das informações oriundas do campo empírico da pesquisa, emergiram dois temas de análise, considerando a similaridade das informações. O primeiro versa sobre a percepção dos profissionais residentes frente à Gestão Pública dos serviços de Saúde Mental onde estão inseridos e sua influência no processo de trabalho e o segundo aborda sobre o processo de formação do profissional residente frente às vivências de gestão pública em serviços de saúde mental.

\section{Tema 1: Percepção dos profissionais} residentes em relação à gestão pública dos serviços de saúde mental onde estão inseridos e sua influência no processo de trabalho.

As falas dos profissionais residentes apontaram algumas dificuldades na gestão dos serviços de saúde mental em função da mudança frequente tanto da coordenação de saúde do município, ou seja, secretário de Saúde, como da coordenação de saúde mental do município: Esse ano mudou a coordenação de saúde mental, mudou já três vezes em um ano, é muita coisa e, a partir dessas mudanças, muda também os processos de trabalho (R6). Atrasa tudo, tu como coordenador conhece toda a rede, começa a se organizar enquanto gestor, quando isso acontece, tu já sai e entra outro gestor dai ela já tem que conhecer toda a rede de novo para daí começar a se organizar, atrasa tudo, é uma bola de neve (R9).

Os participantes deste estudo trouxeram a importância de o gestor ser um profissional da área da saúde. Além disso, que este conheça as Políticas 
de Saúde, Políticas de Saúde Mental e processo de gestão, para assim, gerir da melhor forma o serviço a que está vinculado. Teria que ter alguma especialização, um conhecimento ou alguma formação sobre saúde mental, conhecimento de leis e portarias, isso auxiliaria na gestão mais qualificada. Muitas vezes o pessoal da coordenação do CAPS vai reivindicar alguma coisa para coordenação da saúde mental e ele se quer sabe que existe essas coisas que eles estão reivindicando, e que é lei (R2).

A pesquisa mostra, a partir da fala dos participantes, a importância de os gestores conhecerem as demandas e as necessidades do serviço, para assim evitar gastos financeiros excessivos. Muitas vezes, pela falta de comunicação no espaço de trabalho, há despesas monetárias desnecessárias. Ainda, há menção de que os gestores ouvem pouco o trabalhador dos serviços e tomam decisões sem discussões coletivas prévias. A gente pede um tipo de alimento e vem outros que a gente nem utiliza aqui, para que litros e litros de óleo se a gente não cozinha, vem o almoço das viandas, seria mais fazer lanche, mas nem açúcar tem, daí a gente compraria o que acha necessário, daí vence, vão fora, são mais gastos (R4). O que a gente sabe de gestão é o que é passado em reunião de equipe, um pouco da gestão aqui do CAPS que seriam os relatórios que o pessoal do CAPS faz e de resto é tudo uma questão que vem de cima para baixo então a gente muito pouco sabe o que está acontecendo (R2).

As falas dos participantes apontaram para a falta de apoio à equipe por parte da gestão, juntamente com a insatisfação de promessas não cumpridas. Teve períodos que nós tivemos que fechar o CAPS, com condições insalubres de trabalho e que a gestão ao invés de apoiar e dar suporte para os serviços, acabou criando uma situação mais angustiante, seja por falha na comunicação, seja por dificuldade de compreender a situação do CAPS de vir até aqui, de caminhar junto com os serviços (R6). A gente percebe que as pactuações que foram feitas, nada se concretizou, primeiro era a história do CAPS ad que ia se tonar um CAPS III, que a gente ia ter para questão do plantão noturno, aí depois a história do residencial terapêutico que o município ia ter e também não foi a diante e depois a história de vim um novo CAPS II, depois de desmembrar o ambulatório em regiões de saúde nas ESFs, nada se concretizava, os planos mudavam sempre, mas nada de efetivo se fazia (R5).

Em contrapartida, uma participante trouxe acerca da presença de diálogo entre a equipe e o gestor em momentos de crise e superlotação do serviço, como um aspecto positivo. O acolhimento está fechado desde o ano passado, a gestão está junto, a gente acordou isso com a gestão, porque a equipe estava adoecida e a gestão viu que nós não tínhamos condições de colocar mais usuários aqui dentro sem prestar no mínimo atendimento médico, psicológico, de grupos, não adianta que esse usuário estar conosco, mas que não consegue ser atendido por nós, isso foi um fator positivo, ai é que está quando essa gestão consegue ouvir esse trabalhador (R6).

Os profissionais residentes reforçaram a importância do acompanhamento dos processos de trabalho, por parte do gestor de saúde mental, bem como de sua participação nas reuniões de equipe, no sentido de compreender a dinâmica da equipe e ouvir os profissionais. O coordenador é novo, eu acho que ela está tentando se inserir nos serviços, acho muito bom ela participar para saber como é, porque só a gente vivenciando para saber como funciona, então ela ir no CAPS está sendo bom porque o CAPS vai solicitar alguma coisa para ela e ela vai poder ver que aquilo é necessário (R11).

Os participantes da pesquisa elencaram a cogestão nos serviços de saúde como importante ferramenta para a gestão. São nesses espaços que o profissional residente possui voz e participa ativamente dos processos de gestão nos locais onde estão inseridos. Aqui no CAPS tem uma gestão compartilhada, é bem organizado, se dividem, um coordenador fica responsável por uma coisa, outro por outra, até nas reuniões se dividem quem pode ir (R4). Tem uma cogestão agora, o pessoal da secretaria disse que tinha que ter uma gestão e cogestão, a equipe se mobilizou fez uma cogestão, são três pessoas, eles nos pedem auxílio algumas vezes para muitas coisas (R2).

Os entrevistados também relataram que a falta de recursos financeiros implica na fragilidade do desenvolvimento de algumas atividades terapêuticas prestadas diretamente com o usuário e sua família. Combinar uma Visita Domiciliar com um usuário, tu não sabe quando vai poder ir, tu não vai poder te programar, tem que esperar chegar terça e ver se não vai ter nada mais importante, se o carro novamente não vai vir (R1). Agora a gente está bem ruim de recurso principalmente para horta, a gente não tem quase nada e isso está vindo a influenciar muito no grupo (R2).

\section{Tema 2: O processo de formação do} profissional residente frente às vivências de gestão pública em serviços de saúde mental

Os participantes elencaram as influências e o aprendizado relacionados à gestão nos serviços de saúde mental do município, nos quais estão inseridos. Vale destacar que os profissionais residentes aprenderam a superar frustrações e a lidar com situações difíceis. $A$ gente precisa de materiais que não são repassados, ai se faz com o que tem, tem que ser criativo, tem que inventar alguma forma de tocar os grupos, substituir uma atividade por outra, de alguma forma tem que tocar o grupo. Muitas pessoas não querem o grupo de conversa, daí tem que pensar em outras possibilidades. Aprendi como que é o trabalho no serviço público, esses impasses que a gente se depara e tem que 
tentar dar uma solução, trabalhar sem tantas possibilidades, acho que foi bom, não sei se em outro lugar vai ser tão diferente, acho que me fortaleceu para trabalhar, para aceitar frustração, acho que me preparo (R5).

Os pesquisados destacaram como aprendizado o amadurecimento pessoal pela inserção na equipe dos serviços, em relação a saber ouvir, tencionar e unir os trabalhadores. No entanto, este é um processo lento que deve ser construído junto com a equipe, nos serviços. A gente tem que saber ouvir os profissionais que trabalham em toda a rede (R4). Tu aprende a tencionar também, não só fazer o que a gestão quer, mas a tencionar, também é uma troca, não só aceitar, precisamos conversar, gestor vem aqui, conversa, a gente está tentando ver se consegue trabalhar um pouco dessa questão, da união, da sensação de pertencimento. A nossa equipe se reúne uma vez por mês só os profissionais, isso foi uma coisa que a residência trouxe para eles (R3).

O profissional residente deve implicar-se com os processos de gestão, para tentar apropriar-se desse conhecimento e ser protagonista de sua própria formação. Tem uns que nunca falam, que passam a reunião inteira como corpo presente, eu acho que isso tem muita implicação, tem pessoas ou tão fazendo residência só por fazer ou porque é uma possibilidade econômica muito boa (R6).

Ainda, os participantes mencionaram que apreenderam uma série de conhecimentos referentes ao processo de gestão, sentindo-se preparados para gerenciar um CAPS. Os mesmos apontaram o Núcleo de Educação Permanente em Saúde e Núcleo de Apoio à Saúde Mental como espaços para fazer gestão. Me sinto preparada para assumir a coordenação do CAPS. Aprendi bastante como se deve fazer gestão e como não deve, tem que ter a participação de todos os profissionais que atuam na ponta (R4). Eu acho que a gente acaba fazendo um pouco de gestão, extrapolando o CAPS, quando a gente consegue participar do Núcleo de Educação Permanente em Saúde, eu acho que ali quando a gente faz capacitação, quando a gente chama os profissionais, eu acho que isso faz parte da gestão, acho que isso é gestão (R6).

Outros participantes do estudo relataram ter adquirido pouco conhecimento acerca da gestão dos serviços e de não ter participado de nenhuma reunião com gestores do município no período de sua formação como residente. Não saio com conhecimento de gestão, de melhores formas de se fazer, porque muito pouco eu tenho acesso a isso (R8). Eu não participo de nenhuma reunião, nada do que eu possa colocar meu ponto de vista, para dizer como que eu acho que tem que ser, acho que residente nenhum participa disso (R9).

Um participante assinalou acerca da importância da participação dos usuários no processo gestão. Isso poderia incentivá-los a reivindicar seus direitos junto aos gestores. Eles ficam indignados, até a gente tenta empoderar eles que é um direito que eles têm, que o serviço tem que receber esses materiais, para tentar eles se mobilizar também (R4).

\section{Discussão}

No contexto da gestão em saúde, o coordenador assume importante papel no processo de trabalho. Entretanto, a gestão não se limita a um único ator, mas engloba uma equipe que possa conciliar projetos e valores com a prática da gestão. Os gestores precisam ter noção que suas atitudes não se restringem em governar, administrar e organizar, mas seu elemento central é o ser humano(11).

Vale destacar também que as mudanças da coordenação de saúde podem trazer prejuízos em função da interrupção de serviços, mas por outro lado podem ser benéficas ao processo de trabalho por possibilitar a inserção de ideias novas e promissoras. Toda organização tem alguma rotatividade de pessoa, mas quando ela implica na perda de pessoas estratégicas, constitui-se em fator de ruptura e prejudica a eficiência organizacional.

Para que a assistência à saúde mental seja qualificada, é necessário que os serviços de saúde se articulem por meio das Redes de Atenção Psicossocial (RAPS), descritas pela Portaria 3.088 de $2011^{(4)}$. Além disso, é relevante que gestores, trabalhadores de saúde e população tenham conhecimento dos avanços referentes à Reforma Psiquiátrica, incluída a legislação, para que seja possível oferecer ao usuário tratamento adequado, de acordo com as políticas que os regem. Investigação realizada com enfermeiros mostra que os profissionais que estudam sua própria realidade de trabalho e, por meio da atualização do conhecimento e da capacitação, aplicam os resultados produzidos nas suas organizações de serviço, melhoram a qualidade da assistência ${ }^{(12)}$

A qualificação dos profissionais gestores é indispensável para que a gestão seja mais efetiva, porém, de acordo com a realidade de muitos municípios, por vezes os cargos de gestão são de caráter políticopartidário, com pouca qualificação para o exercício da função de gestor. Isso pode repercutir negativamente no andamento dos processos de trabalho.

É possível exercer uma gestão de qualidade quando o gestor possui visão compreensiva, orientação ética, sensibilidade e habilidade de comunicação e articulação(13). Compreende-se que, por meio de práticas de escuta, a gestão possa conhecer as necessidades dos serviços substitutivos em saúde 
mental e, assim, proporcioná-los suporte às questões relativas a esses aspectos.

Construir confiança nos processos de gestão implica na capacidade de contar uns com os outros para a resolução de problemas, para compartilhamento de resultados positivos e para adotar a tomada de decisão coletiva. Para que a relação de confiança se estabeleça, é imprescindível a valorização do conhecimento de todos os integrantes da equipe, diálogo e respeito de opiniões. A gestão social na saúde, por meio da escuta qualificada, possibilita a interlocução entre as pessoas, para que a interação ocorra e o coletivo se faça presente ${ }^{(13)}$.

Nos momentos de crises, ressalta-se a importância da proximidade dos gestores para promover o apoio emocional, fortalecer a confiança, discutir novas direções ao serviço e evitar a disrupção da equipe. O apoio da gestão nesses momentos tranquiliza a equipe e também aos usuários, podendo oferecer um serviço de qualidade, o que é fundamental no tratamento às pessoas com transtorno mental.

As reuniões de equipe são importantes ferramentas de trabalho que possibilitam trocas de experiências e conhecimentos entre trabalhadores e gestão. As reuniões horizontalizadas fortalecem a inter-relação da equipe e o trabalho por ela desenvolvido, oferecem suporte para enfrentar os desafios do cotidiano profissional, geram motivações para o trabalho, além de propiciarem a criação de espaços patentes para dividir angústias e sofrimentos. Além disso, relações horizontalizadas podem contribuir na co-reponsabilização dos integrantes da equipe na tomada de decisões e encaminhamentos necessários $^{(14)}$. Esses momentos de troca entre gestor $\mathrm{e}$ profissionais dos serviços de saúde mental, por meio das reuniões de equipe, são importantes dispositivos, com repercussões positivas nas atividades desenvolvidas e, consequentemente, na atenção ao usuário e sua família.

No mesmo sentido, a cogestão pode ser uma estratégia que contribui na gestão dos serviços de saúde mental, pois se constitui em exercício compartilhado de um programa, serviço, sistema ou política social, com a participação de diferentes sujeitos, com distintos interesses e diversas inserções sociais. O exercício compartilhado implica coparticipação de todos na totalidade das etapas do processo de gestão: definição de objetivos e de diretrizes, diagnóstico, interpretação de informações, tomada de decisão e avaliação de resultados $^{(15)}$. A cogestão é uma forma de participação do profissional residente no serviço de saúde mental, que implica diretamente na sua formação.

No cenário da saúde mental, a responsabilidade de concretizar as propostas da Reforma Psiquiátrica, com modificações nas práticas de saúde, por vezes, com características manicomiais, recai sobre os profissionais de saúde que compõem a equipe dos serviços substitutivos em saúde mental. Nesse cenário, a formação de profissionais, por meio de inserção em Residência Multiprofissional em Saúde, pode influenciar positivamente na qualidade da assistência ao usuário. A formação de recursos humanos é importante no contexto do movimento de estruturação das redes de atenção à saúde, pois permite à consolidação de sistemas de saúde integrados que favoreça o acesso com continuidade assistencial, integralidade da atenção e utilização coerente dos recursos existentes ${ }^{(16)}$. Os recursos humanos são elementos essenciais para obtenção de desempenho satisfatória no contexto das Redes de Atenção à Saúde(17). O envolvimento do profissional residente com o serviço, com suas potencialidades e fragilidades, oferta significativa vivência, o que contribui para sua formação, contextualizada e convergente à realidade do ambiente de trabalho.

O diálogo e a aproximação dos trabalhadores são importantes na construção de um novo modo de trabalho em saúde, centrado no usuário, com qualidade, resolubilidade e equidade. As estratégias de formação para o SUS, por meio da Residência Multiprofissional, apresentam potencialidades pedagógicas e políticas para transformação do modelo de atenção e das práticas de cuidado em saúde, a partir da aprendizagem vivenciada no contexto do trabalho nos serviços de saúde ${ }^{(18)}$.

Estudo mostra que a Residência Multiprofissional em Saúde tende a ver o usuário de maneira integral, em seus aspectos biopsicossociais, fato que viabiliza a interlocução entre os profissionais de diferentes áreas, enfatizando assim o trabalho em equipe ${ }^{(19)}$. Os profissionais residentes percebem que, apesar de o trabalho ser realizado em equipe, cada um possui sua individualidade, no sentido de não interferir na atuação do outro, mas contribuindo para o desenvolvimento da prática multiprofissional, em beneficio do paciente ${ }^{(20)}$.

Percebe-se que o profissional residente possui papel importante junto à equipe de saúde do serviço, instigando debates e trazendo referenciais teóricos para a prática cotidiana. De outro modo, o amadurecimento profissional do residente decorre das vivências que os serviços de saúde oferece, neste caso, de saúde mental.

A Residência Multiprofissional tem como objetivo a busca do crescimento profissional do residente e a transformação do serviço de saúde onde este está inserido, por meio do incentivo à reflexão sobre as práticas ali desenvolvidas. No entanto, é preciso estabelecer um perfil de competências voltado ao mundo do trabalho, que contemple as necessidades de saúde 
da população e que contribua para a consolidação do SUS ${ }^{(18)}$. Nesse sentido, o profissional residente possui um papel importante no decorrer de sua formação que é promover mudanças no serviço. Para isso, é necessário a implicação do mesmo em seu ambiente de trabalho.

Pesquisa realizada com profissionais residentes mostra que o Programa de Residência Multiprofissional foi compreendido como uma oportunidade de aprendizado e contato com trabalhadores de diferentes áreas. Isso possibilitou a eles assumir nova conduta na prática cotidiana, em que a assistência aos usuários adquiriu caráter mais humanizado e abrangente, pelo compartilhamento efetivo de conhecimentos específicos de cada área e a participação nas atividades de saúde ${ }^{(20)}$. Nesse cenário, pode-se incluir a gestão dos serviços, constituindo-se em uma área a ser fortalecida, a partir das vivências e discussões cotidianas dos profissionais residentes. O residente que participa desses espaços contribui para a construção de gestão nos serviços de saúde e, em contrapartida, fortalece sua formação profissional.

Formar profissionais para atuar no sistema de saúde é um grande desafio. Os conhecimentos, habilidades e atitudes exigidas dos trabalhadores do SUS modificamse rapidamente, desse modo é indispensável aprender a aprender(21). Desse modo, percebe-se a importância de os profissionais residentes estarem nos serviços, vivenciar as demandas referentes à gestão, refletir sobre esse processo de trabalho e poder aprender e contribuir nas discussões acerca da dinâmica dos serviços. Nesse contexto, destaca-se a implicação do profissional residente nessa temática. Observa-se algumas lacunas teóricas no processo de aprendizagem do residente multiprofissional, entre elas sobre Gestão Pública em Saúde, o que pode levar o profissional residente em formação a não se implicar ou não considerar importante o tema relativo à gestão.

A Residência Multiprofissional em Saúde Mental proporciona o crescimento profissional dentro dos serviços, na lógica de trabalho articulado em redes, nos processos de trabalho e nas inter-relações pessoais entre equipe e usuário. Ela possui papel importante para a formação do profissional no âmbito do SUS. A gestão em saúde faz parte dessa formação e proporcionou visão crítica e reflexiva dos processos de trabalho nos serviços de saúde mental onde os profissionais residentes estavam inseridos, bem como a visão do seu papel nesse contexto.

Ainda, ressalva-se a importância da participação do usuário no processo de gestão. A Portaria Nº 2.488/2011 enfoca a participação dos usuários como forma de ampliar sua autonomia e capacidade na construção do cuidado a sua saúde, tanto no enfrentamento dos determinantes e condicionantes a sua saúde, como na organização e orientação dos serviços de saúde, a partir de práticas centradas no usuário e no exercício do controle social(22). O usuário empoderado torna-se instrumento de mudança para si e para a coletividade, na construção de fazer saúde. Assim, participa do processo de gestão no município onde está inserido.

\section{Considerações Finais}

A pesquisa contemplou os objetivos do estudo. Entende-se que a gestão influencia diretamente nos processos de trabalho em saúde mental, desde o entendimento da gestão frente às Políticas de Saúde Mental como o conhecimento das necessidades e demandas dos serviços. O processo de gestão, por vezes, impossibilita que o profissional residente atue em rede e em atividades direcionadas ao tratamento do usuário. Nesse mesmo contexto, a gestão repercute também nos trabalhadores, produzindo sofrimento e desmotivação, tanto no coletivo como no individual, isto pela falta de apoio ou pelas promessas de mudanças não cumpridas por parte de gestores.

A escuta e o diálogo por parte dos gestores com trabalhadores dos serviços de saúde mental são essenciais para a construção coletiva dos processos de trabalho. Nesse sentido, a cogestão entre as equipes dos serviços, onde o residente possui voz na construção desses espaços, é fundamental para empoderar os profissionais a participar das decisões referente à rede de saúde mental e dos processos de trabalho nesses espaços.

A Residência Multiprofissional é um dispositivo que proporciona ao profissional residente maior aproximação e conhecimento frente aos processos de Gestão Pública em Saúde, pois possibilita que ele se aproprie da dinâmica do serviço e, juntamente com a equipe, construa estratégias de trabalho, que beneficiem a população assistida. A Residência Multiprofissional proporcionou aprendizado e crescimento profissional para a maioria dos entrevistados perante as vivências e experiências em Gestão em Saúde, bem como a necessidade de o residente buscar implicar-se com os processos de gestão do município e dos serviços e empoderar o usuário nessa mesma perspectiva.

\section{Referências}

1. Amarante P. Loucos Pela Vida: a trajetória da Reforma Psiquiátrica no Brasil. $2^{\mathrm{a}}$ edição; $6^{\mathrm{a}}$ reimpressão. Rio de Janeiro: Fiocruz; 2013.

2. Ministério da Saúde (BR). Secretaria de Atenção à Saúde. DAPE. Coordenação Geral de Saúde Mental. Reforma psiquiátrica e política de saúde mental no Brasil. 
Documento apresentado à Conferência Regional de Reforma dos Serviços de Saúde Mental: 15 anos depois de Caracas. OPAS. Brasília, MS; 2005.

3. Ministério da Saúde (BR). Lei № 10.216 de 6 de abril de 2001. Dispõe sobre a proteção e os direitos das pessoas portadoras de transtornos mentais e redireciona o modelo assistencial em saúde mental. Brasília, MS; 2001.

4. Ministério da Saúde (BR). Portaria No 3.088 de 23 de dezembro de 2011. Institui a Rede de Atenção Psicossocial para pessoas com sofrimento ou transtorno mental e com necessidades decorrentes do uso de crack, álcool e outras drogas, no âmbito do Sistema Único de Saúde (SUS). Brasília: MS; 2011.

5. Ministério da Saúde (BR). Portaria Interministerial N. ${ }^{\circ}$ 2.117/05. Institui a residência multiprofissional em Saúde. Brasília: Ministério da Saúde e Ministério da Educação; 2005. 6. Ministério da Saúde (BR). Resolução N. ${ }^{\circ}$ 02/12. Dispõe sobre Diretrizes Gerais para os Programas de Residência Multiprofissional e em Profissional de Saúde. Resolução CNRMS No 2, de 13 de abril de 2012. Diário Oficial da União; Poder Executivo, Brasília: MS; 2012. Seção I, p. 24. 7. Ministério da Saúde (BR). Secretaria de Atenção à Saúde. Política Nacional de Humanização. Formação e intervenção. Cadernos HumanizaSUS; v. 1. Série B. Textos Básicos de Saúde. Brasília: MS; 2010.

8. Righi, LB. Apoio matricial e institucional em Saúde: entrevista com Gastão Wagner de Sousa Campos. Rev. Interface (Botucatu). 2014;18(1): 1145-1150.

9. Minayo MCS. O desafio do conhecimento: pesquisa qualitativa em saúde. 10. ed. São Paulo: Hucitec; 2014.

10. Ministério da Saúde (BR). Resolução N 466/12 do Conselho Nacional de Saúde, de modo a proteger os direitos dos participantes. Brasília: MS; 2012.

11. Bardach E. A practical guide for policy analysis: the eightfold path to more effective problem solving. $4^{\mathrm{a}}$ ed. Washington, D.C.: CQPress; 2012.

12. Ferreira RE, Tavares CMM, Santos GS, Fonseca PIMN. Motivação do enfermeiro para ingressar em uma pósgraduação stricto sensu. Revista Baiana de Enfermagem 2015;29(2):180-185.

13. Raimundo JS, Cadete MMM. Escuta qualificada e gestão social entre os profissionais de saúde. Acta Paul Enferm. 2012:25(2):61-67.

14. Verdi M, Caetano TL, Navarro LM, Trepte RF. A cogestão e a inclusão na formação de apoiadores da Política Nacional de Humanização nos territórios do Rio Grande do Sul, Santa Catarina e São Paulo. Sau. \&Transf. Soc. 2014;5(2):50-58.

15. Cunha GT, Campos GWS. Apoio matricial e atenção primária em saúde. Saude soc.2011;20(4):961-970.

16. Lavras C. Atenção primária à saúde e a organização de redes regionais de atenção à saúde no Brasil. Saude soc. v. 20, n. 4, p. 867-874, 2011.
17. Gonçalves CR, Cruz MT, Oliveira MP, Morais AJD, Moreira K S, Rodrigues CAQ et al. Recursos humanos: fator crítico para as redes de atenção à saúde. Saúde debate. 2014;38(100):26-344.

18. Nascimento DG, Oliveira MAC. Competências profissionais e o processo de formação na residência multiprofissional em Saúde da Família. Saúde Soc. 2010;19(4):814-827.

19. Peres RS, Anjos ACY, Rocha MA, Guimarães AGC, Borges G M, Souza KG et al. O trabalho em equipe no contexto hospitalar: reflexões a partir da experiência de um programa de residência multiprofissional em saúde. Em Extensão. 2011;10(1):113-120.

20. Silva JC, Contim D, Ohl RIB, Chavaglia SRR, Amaral EMS. Percepção dos residentes sobre sua atuação no programa de residência multiprofissional. Acta Paul enferm. 2015:28(2):132-138.

21. Batista KBC, Gonçalves OSJ. Formação dos profissionais de saúde para o SUS: significado e cuidado. Saúde soc. 2011;20(4):884-899.

22. Ministério da Saúde. Portaria №2.488, de 21 de outubro de 2011. Aprova a Política Nacional de Atenção Básica, estabelecendo a revisão de diretrizes e normas para a organização da Atenção Básica, para a Estratégia Saúde da Família (ESF) e o Programa de Agentes Comunitários de Saúde (PACS). Brasília: MS;2011. 